\title{
Detection by Rt-Pcr and Molecular Characterization of Tremovirus A Obtained from Clinical Cases of Avian Encephalomyelitis (AE) Outbreaks in Brazil
}

\section{-Author(s)}

\author{
De la Torre $D^{\prime}$ \\ Nuñez LF' \\ Parra SHS \\ Astolfi-Ferreira CS \\ Ferreira AJPI
}

Department of Pathology, School of Veterinary Medicine, University of São Paulo, São Paulo, SP, 05508-270, Brazil.

\section{Mail Address}

Corresponding author e-mail address Antonio J Piantino Ferreira

Department of Pathology, School of Veterinary Medicine, University of São Paulo, São Paulo, SP, 05508-270, Brazil. Email: ajpferr@usp.br

\section{-Keywords}

Chicken, Tremovirus A, Avian

Encephálomyelitis, detection, molecular characterization.

\section{ABSTRACT}

This study determined the presence of Tremovirus A as the possible agent related to Avian Encephalomyelitis in broiler chicks from the states of São Paulo (SP) Paraná (PR), Goiás (GO), Santa Catarina (SC) and Rio Grande do Sul (RS), between the years 2006 and 2015. Samples of the nervous, digestive, respiratory, immune, and renal systems, plus muscular organs from broiler chicks with neurological problems such as ataxia and muscle tremors, and four (4) commercial vaccines as positive control, were tested by reverse-transcriptase (RT-PCR) amplification and DNA sequencing. A highly conserved region (P1) of the viral genome, was used to amplify a segment which encodes a structural protein VP4. Out of 112 samples, 46 were positive (42\%) for Tremovirus A, that was identified in the nervous, digestive, respiratory, renal and immune systems. The phylogenetic analysis clustered together the nucleotide sequences of the 46 samples, the four commercial vaccine strains and the reference sequence of Calnek strain obtained from the GenBank. According to these results, we conclude that the presence of Tremovirus A in these Brazilian chicken flocks distributed in all states was due to flaws in the biosecurity measurements.

\section{INTRODUCTION}

Tremovirus A, also known as Avian Encephalomyelitis Virus (AEV) until 2014 (Knowles et al., 2012), was described for the first time in the United Stated in 1932 (Jones, 1932) and it has been associated to the Avian Encephalomyelitis (AE) disease (Tannock \& Shafren, 1994). This disease mainly affects young birds like broilers, turkeys, pheasants and quails between 1 or 2 weeks of age (Suarez, 2013). Although adult birds do not show clinical signs of disease (Tannock \& Shafren, 1994), it may decrease egg production and hatchability (Taylor et al., 1955), affecting the poultry industry around the world economically (Calnek, 1998; Bakhshesh et al., 2008). Young birds infected with Tremovirus $A$ have shown signs of ataxia, weakness in the legs, reduced mobility and tremors mainly in the head and neck (Olitsky, 1939). Although the Tremovirus A has a protein similarity with the genus Hepatovirus (Marvil et al., 1999), it is classified within the genus Tremovirus, of the family Picornaviridae (Knowles et al., 2012), and until now this is the only specie in this genus. The viral particle has a diameter of 24-32 nm, non-enveloped, icosahedral and with an external structure composed by 32-42 capsomeres (Gosting et al., 1980). The virus genome contains 7032 bp encoding a polyprotein $P 1, P 2$ and P3. The P1 region codes 4 structural proteins: VP4, VP2 (VP0), VP3 e VP1. The P2 and P3 regions encode the non-structural proteins 2A, 2B e 2C e 3A, 3B, 3C e 3D (Marvil et al., 1999). There 
are two types of strains of Tremovirus A, a natural enterotropic strain and a highly neurotropic embryoadapted strain (Suarez, 2013). The enterotropic strain is transmitted horizontally by the oral route, and it is disseminated by feces (Boros et al., 2014). This strain causes neurological problems in susceptible chicks, and it is practically asymptomatic in birds older than 3-4 weeks of age. However, this virus can cause a drop in egg production in breeders and laying hens, in addition to vertical transmission of the virus through the eggs (Calnek, 1998). The embryo-adapted strain is produced by repeated passages by intracerebral inoculation in chicks or inoculation in embryonated eggs (Tannock \& Shafren, 1994). These strains cause severe neurological changes in birds of any age after intracerebral inoculation and even by parenteral inoculation (Calnek, 1998), but after oral ingestion, the ability to be transmitted by feces is lost due the incapacity of multiplication in the digestive cells of birds (Ikeda et al., 1976). The transmission of enteric strains occurs horizontally through the feces and vertically through infected embryonated eggs, both under natural conditions (Yu et al., 2015). The time of virus excretion depends on the age of the infected birds, so, birds with less than two weeks of age usually excrete the virus for two weeks, and birds older than 3 weeks of age, excrete the virus for about 5 days (Westbury \& Sinkovic, 1978). Vertical transmission occurs when breeders do not show immunological activation against the virus and are infected during the laying period, transmitting the virus to the progeny (Freitas \& Back, 2015). The objective of this work was to describe the several cases of neurological disturbs, associated with Tremovirus A, in chicks in the first week of age and establish a molecular characterizes in comparison with vaccine strains used in Brazil.

\section{MATERIALS AND METHODS}

\section{Field samples}

During the period of 2006 to 2015, the Laboratory of Avian Diseases - School of Veterinary Medicine of the University of São Paulo, received 112 samples comprised of different organs such as nervous, digestive, respiratory, renal and immune systems (cecal tonsils, bursa and thymus) of broilers from 21 poultry farms distributed in five Brazilian states, with history of neurological problems and suspected of Avian Encephalomyelitis, which are in the states of SP $(n=14), \operatorname{PR}(23), \operatorname{RS}(n=45), G O(n=11), S C$ $(n=19)$. These samples belonged to broilers between six and 49 days of age, as described following: whole brain $(n=24)$, intestine $(n=20)$, gizzard $(n=12)$, proventriculus $(n=7)$, sciatic nerve $(n=7)$, liver $(n=7)$, ceca $(n=6)$, lung $(n=5)$, kidney $(n=5)$, bursa $(n=4)$, thymus $(n=3)$, cecal tonsil $(n=2)$, cloacal swab $(n=2)$, trachea $(n=2)$, breast muscle $(n=2)$, pancreas $(n=1)$, spleen $(n=1)$, heart $(n=1)$ and bone marrow $(n=1)$. Each sample was received, macerated and conserved at $-20^{\circ} \mathrm{C}$. Approximately $750 \mu \mathrm{g}$ of the macerated samples were used for RNA extraction. A sample was suspended in Phosphate Buffered Saline (PBS) 0.1M, $\mathrm{pH}$ 7.4, in a 1:1 proportion. The suspensions were vortexed and subjected to three freeze and thaw cycles to release the genetic material from cells. The suspensions were finally centrifugated at $12,000 \times$ $\mathrm{g}$ for 30 minutes at $4^{\circ} \mathrm{C}$, and then an aliquot was collected for RNA extraction. Commercial vaccines from Zoetis, Laboratory Bio-Vet, CEVA and Merial were used as positive controls and for comparison of phylogenetic analysis.

\section{Reverse-transcriptase PCR and DNA sequencing}

Isolation of RNA was performed by the PhenolChloroform method as described by Chomczynski (1987), and the final product was stored at $-20^{\circ} \mathrm{C}$. RNA concentrations were measured on the NanoDrop 2000 Spectrophotometer (ThermoFisher Scientific Inc., Waltham, MA, USA). The final RNA concentration for the Reverse Transcriptase (RT) was approximately $1.500 \mathrm{ng} / \mu \mathrm{l}$. The reaction mix for the RT was developed according to the Moloney Murine Leukemia Virus Reverse Transcriptase (ThermoFisher Scientific Inc., Waltham, MA, USA) kit instructions, using Random Primers synthetized by ThermoFisher Scientific Inc., Waltham, MA, USA. The primers used for the PCR were those described by Xie et al. (2005), MK AE 1 (CTTATGCTGGCCCTGATCGT) e and MK AE 2 (TCCCAAATCCACAAACCTAGCC) which were designed to amplify a 619 bp specific segment of the VP2 gene in the P1 region of the viral genome published by Marvil et al. (1999). The PCR reaction mix contained $2.5 \mu \mathrm{l}$ of $10 \mathrm{X}$ PCR buffer, $4 \mu \mathrm{l}$ of the 1.25 mMdeoxynucleotide triphosphate (dNTPs) mix, $1.25 \mu \mathrm{l}$ of each $10 \mathrm{pmol}$ primers MK AE1 and MK AE2, $0.75 \mu \mathrm{l}$ of $50 \mathrm{mMMgCl}, 1 \cup$ Taq DNAPolymerase (ThermoFisher Scientific Inc., Waltham, MA, USA), 2,5 $\mu \mathrm{l}$ of CDNA, and ultrapure water to reach the volume of $25 \mu \mathrm{l}$. The amplification reaction occurred under the following conditions: a 
thermal cycle of $95^{\circ} \mathrm{C}$ for five minutes, followed by 34 cycles of denaturation at $94^{\circ} \mathrm{C}$ for one minute, annealing at $50^{\circ} \mathrm{C}$ for 30 seconds, extension at $68^{\circ} \mathrm{C}$ for one minute and a final extension at $72^{\circ} \mathrm{C}$ for 10 minutes. The final reaction was stored at $-20^{\circ} \mathrm{C}$. The PCR product was purified using the GPXTM PCR DNA and Gel Band Purification kit (GE Healthcare, Piscataway, NJ, USA), according to the manufacturer's instruction. Each purified sample was sequenced by the Sanger method using the two primers MK AE1 and MK AE2 for the forward and reserve reactions, using the BigDye ${ }^{\circledR}$ Terminator Cycle Sequencing Kit V3.1 (ThermoFisher Scientific Inc., Waltham, $M A, U S A)$. The reactions were analyzed by the $A B I$ 3730 DNA Analyzer (ThermoFisher Scientific Inc., Waltham, MA, USA).

\section{Sequence analysis}

The nucleotide sequences were edited by using the CLC Main Work Bench 7.7.1, software (CLC Bio-Qiagen, Aarhus, Denmark) and aligned with reference sequences: enterotropic strain Calnek (NC_003990), embryo-adapted strain Van Roekel (AY517471), Chinese strain L2Z (AY275539) and strain HM-175 from Hepatovirusgenus (NC_001489). The alignment was performed using the CLUSTAL $W$ method, available in the ClustalX 2.0 software (EMBL-EBI, Wellcome Genome Campus, Hinxton, Cambridgeshire, UK). The phylogenetic tree was inferred using Neighbor-Joining statistical method with integrated in the MEGA 7.0.18 software (Tamura et al., 2011). The similarity matrix for nucleotides and amino acids, were generated in the BioEdit Sequence Alignment Editor v. 7.2.5 software (BioEdit Sequence Alignment Editor v. 7.2.5 software).

\section{RESULTS}

\section{Reverse-Transcriptase -PCR}

From 112 samples tested with RT-PCR, the $619 \mathrm{bp}$ specific segment was amplified in 46 samples (42\%) (Table 1). The positive results for each organ were: whole brain 10/24 (41.66\%), intestine 9/20 (45\%), gizzard 5/12 (41.7\%), proventriculus 5/7 (71.4\%), sciatic nerve $5 / 7(71,4 \%)$, liver $4 / 7(57.1 \%)$, ceca $3 / 6$ $(50 \%)$, lung $2 / 5(40 \%)$, kidney $2 / 5(40 \%)$, Bursa $2 / 4$ $(50 \%)$, thymus $1 / 3(33.3 \%)$, cecal tonsil $1 / 2(50 \%)$ in Table 1. Tremovirus A was not detected in samples of cloaca swabs, pool of enteric and nerve tissues, trachea, breast muscle, pancreas, spleen, heart, and bone marrow.
Table 1 - Percentage of positive and negative results for Tremovirus A, for each group of organs from chickens clinically suspected of Avian Encephalomyelitis.

\begin{tabular}{|c|c|c|}
\hline \multirow{2}{*}{ Sample organs/number } & \multicolumn{2}{|c|}{$\begin{array}{l}\text { Number of organ samples that were } \\
\text { considered positive or negative in RT-PCR }\end{array}$} \\
\hline & $\begin{array}{c}\text { Number of positive/ } \\
\text { percentage }\end{array}$ & $\begin{array}{c}\text { Number of negative/ } \\
\text { percentage }\end{array}$ \\
\hline Whole brain $n=24$ & $10(41.66 \%)$ & $14(58.33 \%)$ \\
\hline Intestine $n=20$ & $9(45 \%)$ & $11(55 \%)$ \\
\hline Gizzard $n=12$ & $5(41.7 \%)$ & $7(58.3 \%)$ \\
\hline Proventriculus $n=7$ & $5(71.4 \%)$ & $2(28.6 \%)$ \\
\hline Sciatic nerve $n=7$ & $5(71.4 \%)$ & $2(28.6 \%)$ \\
\hline Liver $n=7$ & $4(57.1 \%)$ & $3(42.9 \%)$ \\
\hline Ceca $n=6$ & $3(50 \%)$ & $3(50 \%)$ \\
\hline Lung $n=5$ & $2(40 \%)$ & $3(60 \%)$ \\
\hline Kidney $n=5$ & $2(40 \%)$ & $3(60 \%)$ \\
\hline Bursa $n=4$ & $2(50 \%)$ & $2(50 \%)$ \\
\hline Thymus $n=3$ & $1(33.3 \%)$ & $2(66.7 \%)$ \\
\hline Cecal tonsils $n=2$ & $1(50 \%)$ & $1(50 \%)$ \\
\hline Cloaca (swab) n= 2 & $0(0 \%)$ & $2(100 \%)$ \\
\hline Trachea $n=2$ & $0(0 \%)$ & $2(100 \%)$ \\
\hline Breast muscle $n=2$ & $0(0 \%)$ & $2(100 \%)$ \\
\hline Pancreas $n=1$ & $0(0 \%)$ & $1(100 \%)$ \\
\hline Spleen $n=1$ & $0(0 \%)$ & $1(100 \%)$ \\
\hline Heart $n=1$ & $0(0 \%)$ & $1(100 \%)$ \\
\hline Bone marrow $n=1$ & $0(0 \%)$ & $1(100 \%)$ \\
\hline Total $n=112$ & $46(41.1 \%)$ & $66(58.9 \%)$ \\
\hline
\end{tabular}

\section{Sequence analysis}

From 46 samples positive for Tremovirus A, a total of 38 were sequenced by Sanger method due to the optimal concentration of DNA, besides the 4 vaccines used as positive control. The similarity matrix showed that the nucleotide sequences of samples 529-1, 529-3, 529-4, 529-5 and 529-6 had 99.4\% similarity with the nucleotide sequences of the four vaccines and the Calnek strain, $94.7 \%$ with the Van Roekel and L2Z strains, and finally $60.2 \%$ with the HM 175 strain (Table 2). The nucleotide sequences of the remaining 33 samples had $100 \%$ similarity to the nucleotide sequences of the four vaccines and the Calnek strain, 95.2\% with the Van Roekel and L2Z strains, and finally $60.1 \%$ with the HM 175 strain. Regarding the amino acid sequences, the sequences of the 38 samples had $100 \%$ similarity with the four vaccine sequences and the Calnek strain, 98.9\% with the Van Roekel strain, 97.4\% with the L2K strain and 55.8\% with the HM 175 strain. The sequences obtained from this study were submitted to the GenBank with the following accession codes: USPBR-463-3 (KY292311), USP-BR-463-4(KY292312), USP-BR-463-5(KY292313), USP-BR-463-6(KY292314), USP-BR-463-7(KY292315), USP-BR-463-8(KY292316), USP-BR-463-9 (KY292317), USP-BR-463-10 (KY292318), USP-BR-463-11 (KY292319), USP- 
Table 2 - Results of similarity between amino acid and nucleotide sequences of the 38 positive samples, vaccines sequences and the reference sequences of Calnek, Van Roekel, L2K and HM 175 strains. Upper right rows correspond to the amino acid sequences, and lower left rows correspond to the nucleotide sequences. (Part 1)

AMINO ACID SEQUENCES

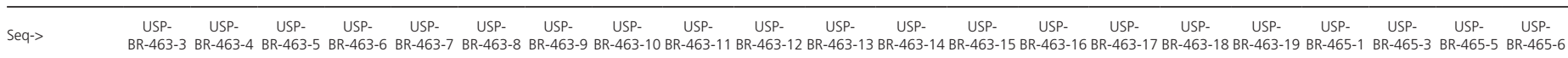
\begin{tabular}{llllllllllllllllllllllll}
\hline USP-BR-463-3 & ID & $100,0 \%$ & $100,0 \%$ & $100,0 \%$ & $100,0 \%$ & $100,0 \%$ & $100,0 \%$ & $100,0 \%$ & $100,0 \%$ & $100,0 \%$ & $100,0 \%$ & $100,0 \%$ & $100,0 \%$ & $100,0 \%$ & $100,0 \%$ & $100,0 \%$ & $100,0 \%$ & $100,0 \%$ & $100,0 \%$ & $100,0 \%$ & $100,0 \%$ \\
\hline
\end{tabular} \begin{tabular}{llllllllllllllllllllll}
\hline USP-BR-463-4 & $100,0 \%$ & ID & $100,0 \%$ & $100,0 \%$ & $100,0 \%$ & $100,0 \%$ & $100,0 \%$ & $100,0 \%$ & $100,0 \%$ & $100,0 \%$ & $100,0 \%$ & $100,0 \%$ & $100,0 \%$ & $100,0 \%$ & $100,0 \%$ & $100,0 \%$ & $100,0 \%$ & $100,0 \%$ & $100,0 \%$ & $100,0 \%$ & $100,0 \%$ \\
\hline
\end{tabular} \begin{tabular}{lllllllllllllllllllllll}
\hline USP-BR-463-5 & $100,0 \%$ & $100,0 \%$ & ID & $100,0 \%$ & $100,0 \%$ & $100,0 \%$ & $100,0 \%$ & $100,0 \%$ & $100,0 \%$ & $100,0 \%$ & $100,0 \%$ & $100,0 \%$ & $100,0 \%$ & $100,0 \%$ & $100,0 \%$ & $100,0 \%$ & $100,0 \%$ & $100,0 \%$ & $100,0 \%$ & $100,0 \%$ & $100,0 \%$
\end{tabular}

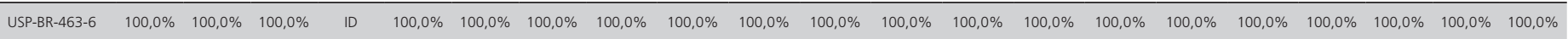
\begin{tabular}{lllllllllllllllllllllllll}
\hline USP-BR-463-7 & $100,0 \%$ & $100,0 \%$ & $100,0 \%$ & $100,0 \%$ & ID & $100,0 \%$ & $100,0 \%$ & $100,0 \%$ & $100,0 \%$ & $100,0 \%$ & $100,0 \%$ & $100,0 \%$ & $100,0 \%$ & $100,0 \%$ & $100,0 \%$ & $100,0 \%$ & $100,0 \%$ & $100,0 \%$ & $100,0 \%$ & $100,0 \%$ & $100,0 \%$ \\
\hline
\end{tabular}

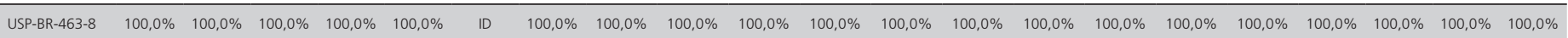
\begin{tabular}{llllllllllllllllllllllll}
\hline USP-BR-463-9 & $100,0 \%$ & $100,0 \%$ & $100,0 \%$ & $100,0 \%$ & $100,0 \%$ & $100,0 \%$ & ID & $100,0 \%$ & $100,0 \%$ & $100,0 \%$ & $100,0 \%$ & $100,0 \%$ & $100,0 \%$ & $100,0 \%$ & $100,0 \%$ & $100,0 \%$ & $100,0 \%$ & $100,0 \%$ & $100,0 \%$ & $100,0 \%$ & $100,0 \%$ \\
\hline
\end{tabular} \begin{tabular}{lllllllllllllllllllllll}
\hline USP-BR-463-10 & $100,0 \%$ & $100,0 \%$ & $100,0 \%$ & $100,0 \%$ & $100,0 \%$ & $100,0 \%$ & $100,0 \%$ & ID & $100,0 \%$ & $100,0 \%$ & $100,0 \%$ & $100,0 \%$ & $100,0 \%$ & $100,0 \%$ & $100,0 \%$ & $100,0 \%$ & $100,0 \%$ & $100,0 \%$ & $100,0 \%$ & $100,0 \%$ & $100,0 \%$ \\
\hline
\end{tabular}

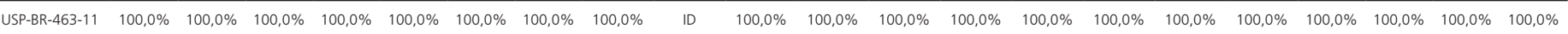

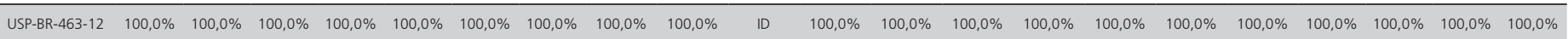
\begin{tabular}{llllllllllllllllllllllll}
\hline USP-BR-463-13 & $100,0 \%$ & $100,0 \%$ & $100,0 \%$ & $100,0 \%$ & $100,0 \%$ & $100,0 \%$ & $100,0 \%$ & $100,0 \%$ & $100,0 \%$ & $100,0 \%$ & ID & $100,0 \%$ & $100,0 \%$ & $100,0 \%$ & $100,0 \%$ & $100,0 \%$ & $100,0 \%$ & $100,0 \%$ & $100,0 \%$ & $100,0 \%$ & $100,0 \%$ \\
\hline
\end{tabular}

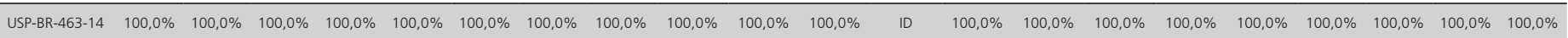
\begin{tabular}{llllllllllllllllllllllll} 
USP-BR-463-15 & $100,0 \%$ & $100,0 \%$ & $100,0 \%$ & $100,0 \%$ & $100,0 \%$ & $100,0 \%$ & $100,0 \%$ & $100,0 \%$ & $100,0 \%$ & $100,0 \%$ & $100,0 \%$ & $100,0 \%$ & ID & $100,0 \%$ & $100,0 \%$ & $100,0 \%$ & $100,0 \%$ & $100,0 \%$ & $100,0 \%$ & $100,0 \%$ & $100,0 \%$ \\
\hline
\end{tabular}

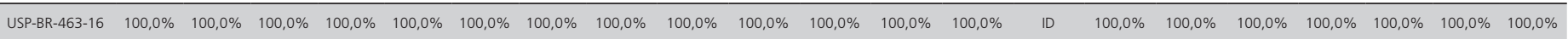
\begin{tabular}{llllllllllllllllllllll}
\hline USP-BR-463-17 & $100,0 \%$ & $100,0 \%$ & $100,0 \%$ & $100,0 \%$ & $100,0 \%$ & $100,0 \%$ & $100,0 \%$ & $100,0 \%$ & $100,0 \%$ & $100,0 \%$ & $100,0 \%$ & $100,0 \%$ & $100,0 \%$ & $100,0 \%$ & ID & $100,0 \%$ & $100,0 \%$ & $100,0 \%$ & $100,0 \%$ & $100,0 \%$ & $100,0 \%$ \\
\hline
\end{tabular}

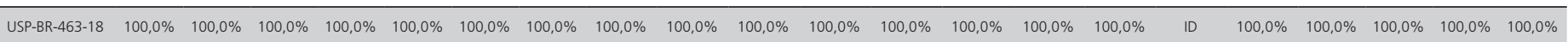

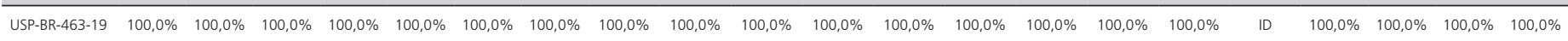

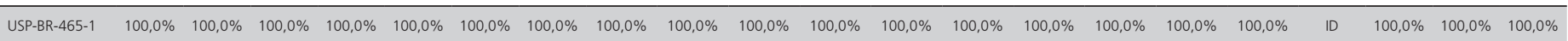
\begin{tabular}{llllllllllllllllllllllll}
\hline USP-BR-465-3 & $100,0 \%$ & $100,0 \%$ & $100,0 \%$ & $100,0 \%$ & $100,0 \%$ & $100,0 \%$ & $100,0 \%$ & $100,0 \%$ & $100,0 \%$ & $100,0 \%$ & $100,0 \%$ & $100,0 \%$ & $100,0 \%$ & $100,0 \%$ & $100,0 \%$ & $100,0 \%$ & $100,0 \%$ & $100,0 \%$ & $1 D$ & $100,0 \%$ & $100,0 \%$ \\
\hline
\end{tabular}

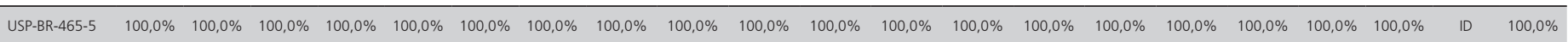

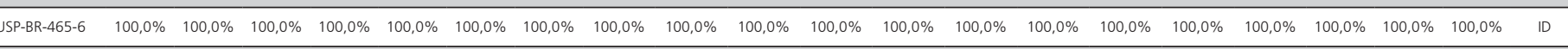

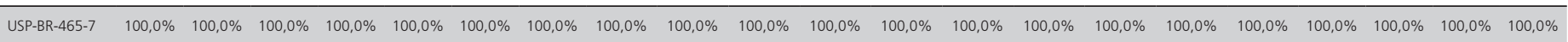

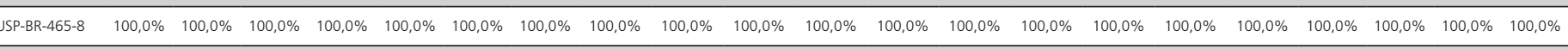

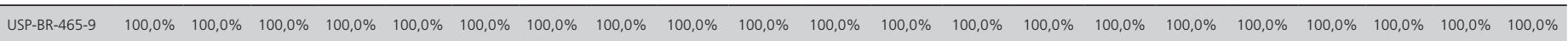

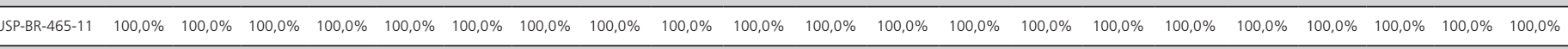

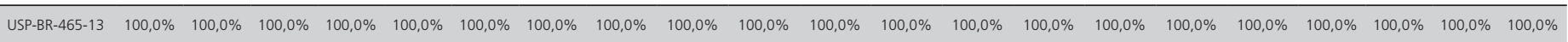
\begin{tabular}{llllllllllllllllllllll}
\hline USP-BR-465-16 & $100,0 \%$ & $100,0 \%$ & $100,0 \%$ & $100,0 \%$ & $100,0 \%$ & $100,0 \%$ & $100,0 \%$ & $100,0 \%$ & $100,0 \%$ & $100,0 \%$ & $100,0 \%$ & $100,0 \%$ & $100,0 \%$ & $100,0 \%$ & $100,0 \%$ & $100,0 \%$ & $100,0 \%$ & $100,0 \%$ & $100,0 \%$ & $100,0 \%$ & $100,0 \%$ \\
\hline USP-BR-
\end{tabular}

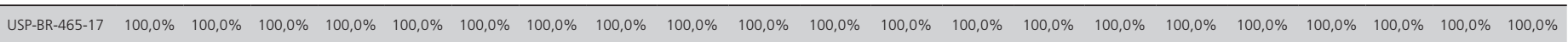
\begin{tabular}{llllllllllllllllllllllll}
\hline USP-BR-465-18 & $100,0 \%$ & $100,0 \%$ & $100,0 \%$ & $100,0 \%$ & $100,0 \%$ & $100,0 \%$ & $100,0 \%$ & $100,0 \%$ & $100,0 \%$ & $100,0 \%$ & $100,0 \%$ & $100,0 \%$ & $100,0 \%$ & $100,0 \%$ & $100,0 \%$ & $100,0 \%$ & $100,0 \%$ & $100,0 \%$ & $100,0 \%$ & $100,0 \%$ & $100,0 \%$ \\
\hline USPR
\end{tabular}

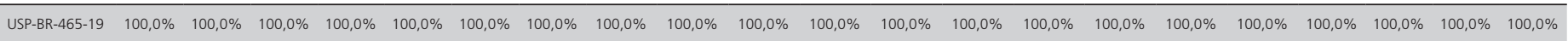

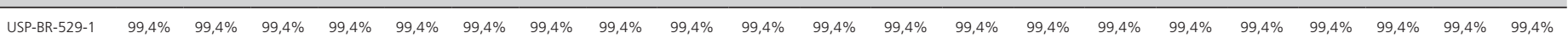

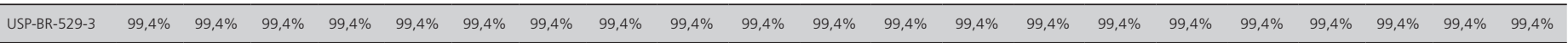
\begin{tabular}{llllllllllllllllllllll}
\hline USP-BR-529-4 & $99,4 \%$ & $99,4 \%$ & $99,4 \%$ & $99,4 \%$ & $99,4 \%$ & $99,4 \%$ & $99,4 \%$ & $99,4 \%$ & $99,4 \%$ & $99,4 \%$ & $99,4 \%$ & $99,4 \%$ & $99,4 \%$ & $99,4 \%$ & $99,4 \%$ & $99,4 \%$ & $99,4 \%$ & $99,4 \%$ & $99,4 \%$ & $99,4 \%$ & $99,4 \%$ \\
\hline
\end{tabular}

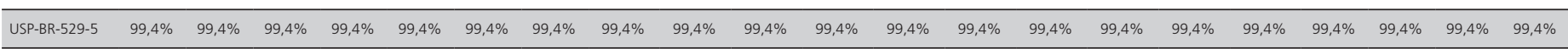

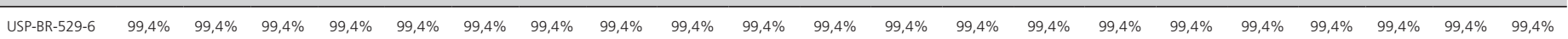
\begin{tabular}{llllllllllllllllllllll}
\hline USP-BR-604-7 & $100,0 \%$ & $100,0 \%$ & $100,0 \%$ & $100,0 \%$ & $100,0 \%$ & $100,0 \%$ & $100,0 \%$ & $100,0 \%$ & $100,0 \%$ & $100,0 \%$ & $100,0 \%$ & $100,0 \%$ & $100,0 \%$ & $100,0 \%$ & $100,0 \%$ & $100,0 \%$ & $100,0 \%$ & $100,0 \%$ & $100,0 \%$ & $100,0 \%$ & $100,0 \%$ \\
\hline
\end{tabular} \begin{tabular}{llllllllllllllllllllllll}
\hline USP-BR-604-8 & $100,0 \%$ & $100,0 \%$ & $100,0 \%$ & $100,0 \%$ & $100,0 \%$ & $100,0 \%$ & $100,0 \%$ & $100,0 \%$ & $100,0 \%$ & $100,0 \%$ & $100,0 \%$ & $100,0 \%$ & $100,0 \%$ & $100,0 \%$ & $100,0 \%$ & $100,0 \%$ & $100,0 \%$ & $100,0 \%$ & $100,0 \%$ & $100,0 \%$ & $100,0 \%$ \\
\hline
\end{tabular}

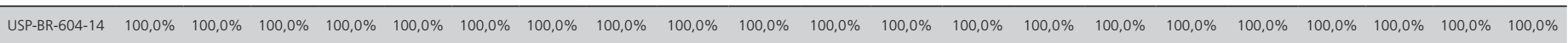

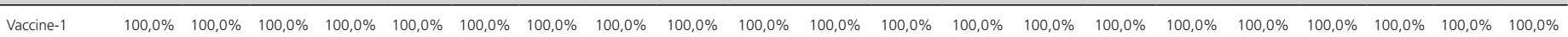

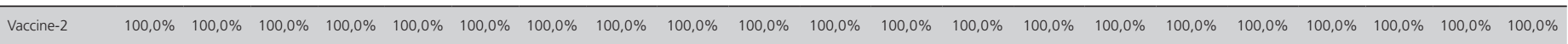
\begin{tabular}{llllllllllllllllllllllll}
\hline Vaccine-3 & $100,0 \%$ & $100,0 \%$ & $100,0 \%$ & $100,0 \%$ & $100,0 \%$ & $100,0 \%$ & $100,0 \%$ & $100,0 \%$ & $100,0 \%$ & $100,0 \%$ & $100,0 \%$ & $100,0 \%$ & $100,0 \%$ & $100,0 \%$ & $100,0 \%$ & $100,0 \%$ & $100,0 \%$ & $100,0 \%$ & $100,0 \%$ & $100,0 \%$ & $100,0 \%$ \\
\hline
\end{tabular}

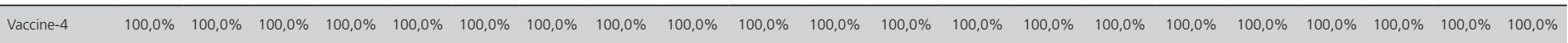
\begin{tabular}{llllllllllllllllllllllll}
\hline Calnek & $100,0 \%$ & $100,0 \%$ & $100,0 \%$ & $100,0 \%$ & $100,0 \%$ & $100,0 \%$ & $100,0 \%$ & $100,0 \%$ & $100,0 \%$ & $100,0 \%$ & $100,0 \%$ & $100,0 \%$ & $100,0 \%$ & $100,0 \%$ & $100,0 \%$ & $100,0 \%$ & $100,0 \%$ & $100,0 \%$ & $100,0 \%$ & $100,0 \%$ & $100,0 \%$ \\
\hline
\end{tabular}

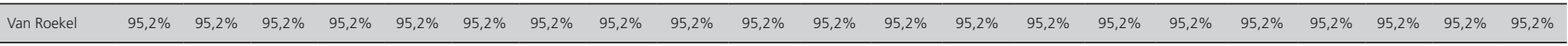
\begin{tabular}{llllllllllllllllllllll}
\hline L2Z & $95,2 \%$ & $95,2 \%$ & $95,2 \%$ & $95,2 \%$ & $95,2 \%$ & $95,2 \%$ & $95,2 \%$ & $95,2 \%$ & $95,2 \%$ & $95,2 \%$ & $95,2 \%$ & $95,2 \%$ & $95,2 \%$ & $95,2 \%$ & $95,2 \%$ & $95,2 \%$ & $95,2 \%$ & $95,2 \%$ & $95,2 \%$ & $95,2 \%$ & $95,2 \%$ \\
\hline
\end{tabular}

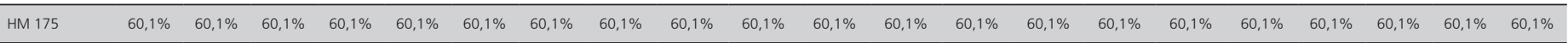


Table 2 - Results of similarity between amino acid and nucleotide sequences of the 38 positive samples, vaccines sequences and the reference sequences of Calnek, Van Roekel, L2K and HM 175 strains. Upper right rows correspond to the amino acid sequences, and lower left rows correspond to the nucleotide sequences. (Part 2)

AMINO ACID SEQUENCES

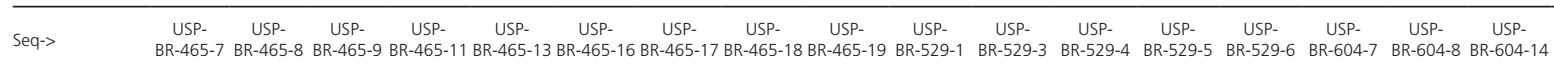

\begin{tabular}{lllllllllllllllllllllll}
\hline USP-BR-463-3 & $100,0 \%$ & $100,0 \%$ & $100,0 \%$ & $100,0 \%$ & $100,0 \%$ & $100,0 \%$ & $100,0 \%$ & $100,0 \%$ & $100,0 \%$ & $100,0 \%$ & $100,0 \%$ & $100,0 \%$ & $100,0 \%$ & $100,0 \%$ & $100,0 \%$ & $100,0 \%$ & $100,0 \%$ & $100,0 \%$ & $100,0 \%$ & $100,0 \%$ & $100,0 \%$ \\
\hline
\end{tabular} \begin{tabular}{lllllllllllllllllllllll}
\hline USP-BR-463-4 & $100,0 \%$ & $100,0 \%$ & $100,0 \%$ & $100,0 \%$ & $100,0 \%$ & $100,0 \%$ & $100,0 \%$ & $100,0 \%$ & $100,0 \%$ & $100,0 \%$ & $100,0 \%$ & $100,0 \%$ & $100,0 \%$ & $100,0 \%$ & $100,0 \%$ & $100,0 \%$ & $100,0 \%$ & $100,0 \%$ & $100,0 \%$ & $100,0 \%$ & $100,0 \%$ \\
\hline
\end{tabular} \begin{tabular}{lllllllllllllllllllllll}
\hline USP-BR-463-5 & $100,0 \%$ & $100,0 \%$ & $100,0 \%$ & $100,0 \%$ & $100,0 \%$ & $100,0 \%$ & $100,0 \%$ & $100,0 \%$ & $100,0 \%$ & $100,0 \%$ & $100,0 \%$ & $100,0 \%$ & $100,0 \%$ & $100,0 \%$ & $100,0 \%$ & $100,0 \%$ & $100,0 \%$ & $100,0 \%$ & $100,0 \%$ & $100,0 \%$ & $100,0 \%$ \\
\hline
\end{tabular} \begin{tabular}{lllllllllllllllllllllll}
\hline USP-BR-463-6 & $100,0 \%$ & $100,0 \%$ & $100,0 \%$ & $100,0 \%$ & $100,0 \%$ & $100,0 \%$ & $100,0 \%$ & $100,0 \%$ & $100,0 \%$ & $100,0 \%$ & $100,0 \%$ & $100,0 \%$ & $100,0 \%$ & $100,0 \%$ & $100,0 \%$ & $100,0 \%$ & $100,0 \%$ & $100,0 \%$ & $100,0 \%$ & $100,0 \%$ & $100,0 \%$ \\
\hline
\end{tabular} \begin{tabular}{llllllllllllllllllllll}
\hline USP-BR-463-7 & $100,0 \%$ & $100,0 \%$ & $100,0 \%$ & $100,0 \%$ & $100,0 \%$ & $100,0 \%$ & $100,0 \%$ & $100,0 \%$ & $100,0 \%$ & $100,0 \%$ & $100,0 \%$ & $100,0 \%$ & $100,0 \%$ & $100,0 \%$ & $100,0 \%$ & $100,0 \%$ & $100,0 \%$ & $100,0 \%$ & $100,0 \%$ & $100,0 \%$ & $100,0 \%$ \\
\hline
\end{tabular}

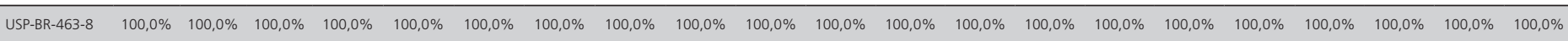
\begin{tabular}{llllllllllllllllllllllll}
\hline USP-BR-463-9 & $100,0 \%$ & $100,0 \%$ & $100,0 \%$ & $100,0 \%$ & $100,0 \%$ & $100,0 \%$ & $100,0 \%$ & $100,0 \%$ & $100,0 \%$ & $100,0 \%$ & $100,0 \%$ & $100,0 \%$ & $100,0 \%$ & $100,0 \%$ & $100,0 \%$ & $100,0 \%$ & $100,0 \%$ & $100,0 \%$ & $100,0 \%$ & $100,0 \%$ & $100,0 \%$ \\
\hline
\end{tabular} \begin{tabular}{llllllllllllllllllllll}
\hline USP-BR-463-10 & $100,0 \%$ & $100,0 \%$ & $100,0 \%$ & $100,0 \%$ & $100,0 \%$ & $100,0 \%$ & $100,0 \%$ & $100,0 \%$ & $100,0 \%$ & $100,0 \%$ & $100,0 \%$ & $100,0 \%$ & $100,0 \%$ & $100,0 \%$ & $100,0 \%$ & $100,0 \%$ & $100,0 \%$ & $100,0 \%$ & $100,0 \%$ & $100,0 \%$ & $100,0 \%$ \\
\hline
\end{tabular}

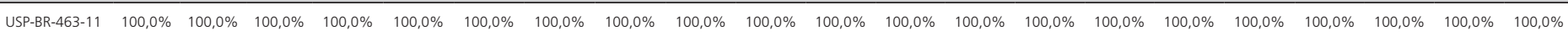
\begin{tabular}{llllllllllllllllllllll}
\hline USP-BR-463-12 & $100,0 \%$ & $100,0 \%$ & $100,0 \%$ & $100,0 \%$ & $100,0 \%$ & $100,0 \%$ & $100,0 \%$ & $100,0 \%$ & $100,0 \%$ & $100,0 \%$ & $100,0 \%$ & $100,0 \%$ & $100,0 \%$ & $100,0 \%$ & $100,0 \%$ & $100,0 \%$ & $100,0 \%$ & $100,0 \%$ & $100,0 \%$ & $100,0 \%$ & $100,0 \%$ \\
\hline
\end{tabular}

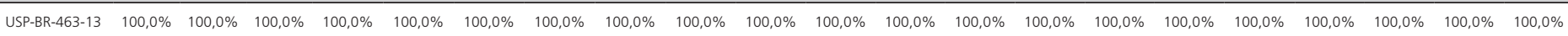
\begin{tabular}{llllllllllllllllllllll}
\hline USP-BR-463-14 & $100,0 \%$ & $100,0 \%$ & $100,0 \%$ & $100,0 \%$ & $100,0 \%$ & $100,0 \%$ & $100,0 \%$ & $100,0 \%$ & $100,0 \%$ & $100,0 \%$ & $100,0 \%$ & $100,0 \%$ & $100,0 \%$ & $100,0 \%$ & $100,0 \%$ & $100,0 \%$ & $100,0 \%$ & $100,0 \%$ & $100,0 \%$ & $100,0 \%$ & $100,0 \%$ \\
\hline
\end{tabular} \begin{tabular}{lllllllllllllllllllllll}
\hline USP-BR-463-15 & $100,0 \%$ & $100,0 \%$ & $100,0 \%$ & $100,0 \%$ & $100,0 \%$ & $100,0 \%$ & $100,0 \%$ & $100,0 \%$ & $100,0 \%$ & $100,0 \%$ & $100,0 \%$ & $100,0 \%$ & $100,0 \%$ & $100,0 \%$ & $100,0 \%$ & $100,0 \%$ & $100,0 \%$ & $100,0 \%$ & $100,0 \%$ & $100,0 \%$ & $100,0 \%$ \\
\hline
\end{tabular} \begin{tabular}{|llllllllllllllllllllllll}
\hline USP-BR-463-16 & $100,0 \%$ & $100,0 \%$ & $100,0 \%$ & $100,0 \%$ & $100,0 \%$ & $100,0 \%$ & $100,0 \%$ & $100,0 \%$ & $100,0 \%$ & $100,0 \%$ & $100,0 \%$ & $100,0 \%$ & $100,0 \%$ & $100,0 \%$ & $100,0 \%$ & $100,0 \%$ & $100,0 \%$ & $100,0 \%$ & $100,0 \%$ & $100,0 \%$ & $100,0 \%$ \\
\hline
\end{tabular} \begin{tabular}{llllllllllllllllllllllll}
\hline USP-BR-463-17 & $100,0 \%$ & $100,0 \%$ & $100,0 \%$ & $100,0 \%$ & $100,0 \%$ & $100,0 \%$ & $100,0 \%$ & $100,0 \%$ & $100,0 \%$ & $100,0 \%$ & $100,0 \%$ & $100,0 \%$ & $100,0 \%$ & $100,0 \%$ & $100,0 \%$ & $100,0 \%$ & $100,0 \%$ & $100,0 \%$ & $100,0 \%$ & $100,0 \%$ & $100,0 \%$ \\
\hline
\end{tabular} \begin{tabular}{llllllllllllllllllllll}
\hline USP-BR-463-18 & $100,0 \%$ & $100,0 \%$ & $100,0 \%$ & $100,0 \%$ & $100,0 \%$ & $100,0 \%$ & $100,0 \%$ & $100,0 \%$ & $100,0 \%$ & $100,0 \%$ & $100,0 \%$ & $100,0 \%$ & $100,0 \%$ & $100,0 \%$ & $100,0 \%$ & $100,0 \%$ & $100,0 \%$ & $100,0 \%$ & $100,0 \%$ & $100,0 \%$ & $100,0 \%$ \\
\hline
\end{tabular} \begin{tabular}{llllllllllllllllllllll}
\hline USP-BR-463-19 & $100,0 \%$ & $100,0 \%$ & $100,0 \%$ & $100,0 \%$ & $100,0 \%$ & $100,0 \%$ & $100,0 \%$ & $100,0 \%$ & $100,0 \%$ & $100,0 \%$ & $100,0 \%$ & $100,0 \%$ & $100,0 \%$ & $100,0 \%$ & $100,0 \%$ & $100,0 \%$ & $100,0 \%$ & $100,0 \%$ & $100,0 \%$ & $100,0 \%$ & $100,0 \%$ \\
\hline
\end{tabular} \begin{tabular}{llllllllllllllllllllllll}
\hline USP-BR-465-1 & $100,0 \%$ & $100,0 \%$ & $100,0 \%$ & $100,0 \%$ & $100,0 \%$ & $100,0 \%$ & $100,0 \%$ & $100,0 \%$ & $100,0 \%$ & $100,0 \%$ & $100,0 \%$ & $100,0 \%$ & $100,0 \%$ & $100,0 \%$ & $100,0 \%$ & $100,0 \%$ & $100,0 \%$ & $100,0 \%$ & $100,0 \%$ & $100,0 \%$ & $100,0 \%$ \\
\hline
\end{tabular}

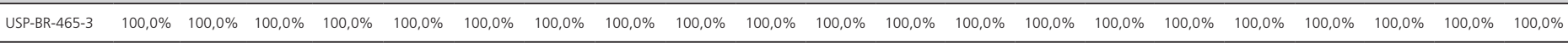
\begin{tabular}{llllllllllllllllllllllll}
\hline USP-BR-465-5 & $100,0 \%$ & $100,0 \%$ & $100,0 \%$ & $100,0 \%$ & $100,0 \%$ & $100,0 \%$ & $100,0 \%$ & $100,0 \%$ & $100,0 \%$ & $100,0 \%$ & $100,0 \%$ & $100,0 \%$ & $100,0 \%$ & $100,0 \%$ & $100,0 \%$ & $100,0 \%$ & $100,0 \%$ & $100,0 \%$ & $100,0 \%$ & $100,0 \%$ & $100,0 \%$ \\
\hline
\end{tabular}

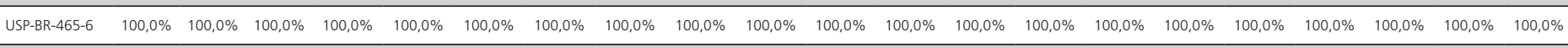
\begin{tabular}{llllllllllllllllllllllll}
\hline USP-BR-465-7 & ID & $100,0 \%$ & $100,0 \%$ & $100,0 \%$ & $100,0 \%$ & $100,0 \%$ & $100,0 \%$ & $100,0 \%$ & $100,0 \%$ & $100,0 \%$ & $100,0 \%$ & $100,0 \%$ & $100,0 \%$ & $100,0 \%$ & $100,0 \%$ & $100,0 \%$ & $100,0 \%$ & $100,0 \%$ & $100,0 \%$ & $100,0 \%$ & $100,0 \%$ \\
\hline
\end{tabular}

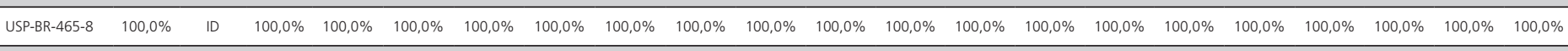
\begin{tabular}{llllllllllllllllllllll}
\hline USP-BR-465-9 & $100,0 \%$ & $100,0 \%$ & ID & $100,0 \%$ & $100,0 \%$ & $100,0 \%$ & $100,0 \%$ & $100,0 \%$ & $100,0 \%$ & $100,0 \%$ & $100,0 \%$ & $100,0 \%$ & $100,0 \%$ & $100,0 \%$ & $100,0 \%$ & $100,0 \%$ & $100,0 \%$ & $100,0 \%$ & $100,0 \%$ & $100,0 \%$ & $100,0 \%$ \\
\hline
\end{tabular}

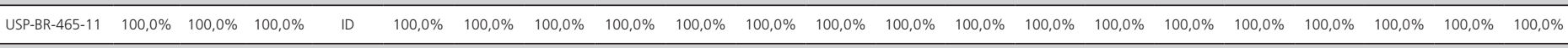

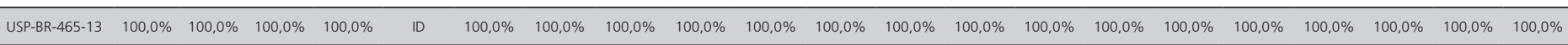

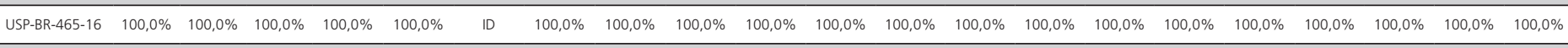
\begin{tabular}{|lllllllllllllllllllllll}
\hline USP-BR-465-17 & $100,0 \%$ & $100,0 \%$ & $100,0 \%$ & $100,0 \%$ & $100,0 \%$ & $100,0 \%$ & ID & $100,0 \%$ & $100,0 \%$ & $100,0 \%$ & $100,0 \%$ & $100,0 \%$ & $100,0 \%$ & $100,0 \%$ & $100,0 \%$ & $100,0 \%$ & $100,0 \%$ & $100,0 \%$ & $100,0 \%$ & $100,0 \%$ & $100,0 \%$ \\
\hline
\end{tabular}

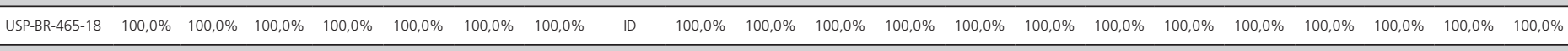
\begin{tabular}{|lllllllllllllllllllllll}
\hline USP-BR-465-19 & $100,0 \%$ & $100,0 \%$ & $100,0 \%$ & $100,0 \%$ & $100,0 \%$ & $100,0 \%$ & $100,0 \%$ & $100,0 \%$ & ID & $100,0 \%$ & $100,0 \%$ & $100,0 \%$ & $100,0 \%$ & $100,0 \%$ & $100,0 \%$ & $100,0 \%$ & $100,0 \%$ & $100,0 \%$ & $100,0 \%$ & $100,0 \%$ & $100,0 \%$ \\
\hline
\end{tabular}

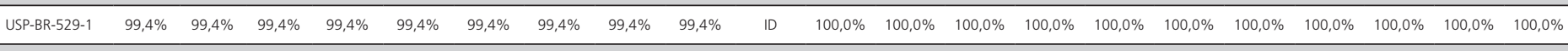
\begin{tabular}{|llllllllllllllllllllll}
\hline USP-BR-529-3 & $99,4 \%$ & $99,4 \%$ & $99,4 \%$ & $99,4 \%$ & $99,4 \%$ & $99,4 \%$ & $99,4 \%$ & $99,4 \%$ & $99,4 \%$ & $100,0 \%$ & ID & $100,0 \%$ & $100,0 \%$ & $100,0 \%$ & $100,0 \%$ & $100,0 \%$ & $100,0 \%$ & $100,0 \%$ & $100,0 \%$ & $100,0 \%$ & $100,0 \%$ \\
\hline
\end{tabular} \begin{tabular}{llllllllllllllllllllllll}
\hline USP-BR-529-4 & $99,4 \%$ & $99,4 \%$ & $99,4 \%$ & $99,4 \%$ & $99,4 \%$ & $99,4 \%$ & $99,4 \%$ & $99,4 \%$ & $99,4 \%$ & $100,0 \%$ & $100,0 \%$ & $1 D$ & $100,0 \%$ & $100,0 \%$ & $100,0 \%$ & $100,0 \%$ & $100,0 \%$ & $100,0 \%$ & $100,0 \%$ & $100,0 \%$ & $100,0 \%$ \\
\hline
\end{tabular}

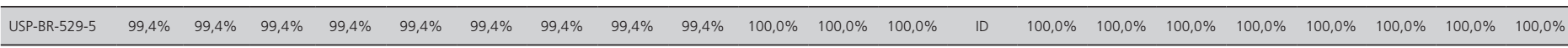
\begin{tabular}{lllllllllllllllllllllll}
\hline USP-BR-529-6 & $99,4 \%$ & $99,4 \%$ & $99,4 \%$ & $99,4 \%$ & $99,4 \%$ & $99,4 \%$ & $99,4 \%$ & $99,4 \%$ & $99,4 \%$ & $100,0 \%$ & $100,0 \%$ & $100,0 \%$ & $100,0 \%$ & ID & $100,0 \%$ & $100,0 \%$ & $100,0 \%$ & $100,0 \%$ & $100,0 \%$ & $100,0 \%$ & $100,0 \%$ \\
\hline USP
\end{tabular}

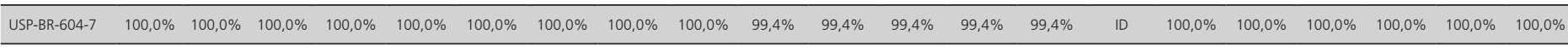
\begin{tabular}{llllllllllllllllllllll}
\hline USP-BR-604-8 & $100,0 \%$ & $100,0 \%$ & $100,0 \%$ & $100,0 \%$ & $100,0 \%$ & $100,0 \%$ & $100,0 \%$ & $100,0 \%$ & $100,0 \%$ & $99,4 \%$ & $99,4 \%$ & $99,4 \%$ & $99,4 \%$ & $99,4 \%$ & $100,0 \%$ & DD & $100,0 \%$ & $100,0 \%$ & $100,0 \%$ & $100,0 \%$ & $100,0 \%$ \\
\hline
\end{tabular}

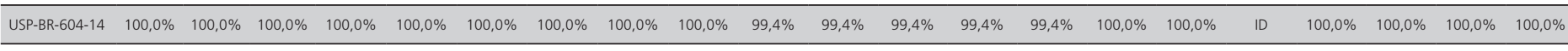

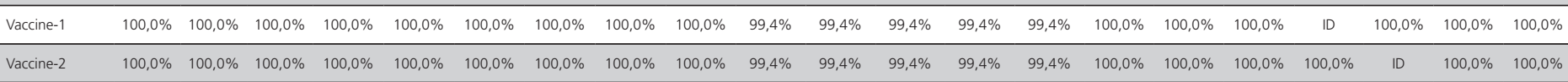

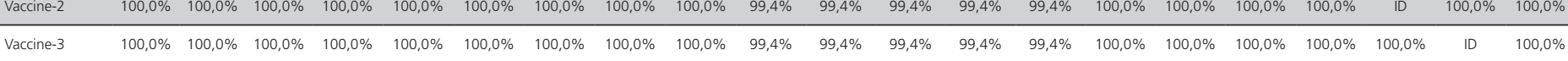

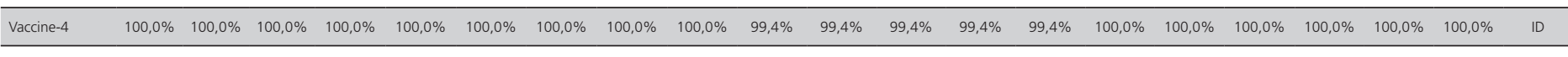
\begin{tabular}{llllllllllllllllllllllll}
\hline Calnek & $100,0 \%$ & $100,0 \%$ & $100,0 \%$ & $100,0 \%$ & $100,0 \%$ & $100,0 \%$ & $100,0 \%$ & $100,0 \%$ & $100,0 \%$ & $99,4 \%$ & $99,4 \%$ & $99,4 \%$ & $99,4 \%$ & $99,4 \%$ & $100,0 \%$ & $100,0 \%$ & $100,0 \%$ & $100,0 \%$ & $100,0 \%$ & $100,0 \%$ & $100,0 \%$ \\
\hline
\end{tabular}

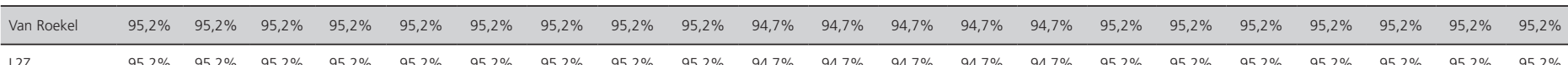

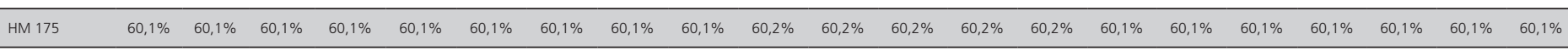


De la Torre D, Nuñez LF, Parra SHS,

Table 2 - Results of similarity between amino acid and nucleotide sequences of the 38 positive samples, vaccines sequences and the reference sequences of Calnek, Van Roekel, L2K and HM 175 strains. Upper right rows correspond to the amino acid sequences, and lower left rows correspond to the nucleotide sequences. (Part 3)

AMINO ACID SEQUENCES

\begin{tabular}{|c|c|c|c|c|}
\hline Seq-> & Calnek & Van Roekel & L2Z & HM 175 \\
\hline USP-BR-463-3 & $100,0 \%$ & $98,9 \%$ & $97,4 \%$ & $55,8 \%$ \\
\hline USP-BR-463-4 & $100,0 \%$ & $98,9 \%$ & $97,4 \%$ & $55,8 \%$ \\
\hline USP-BR-463-5 & $100,0 \%$ & $98,9 \%$ & $97,4 \%$ & $55,8 \%$ \\
\hline USP-BR-463-6 & $100,0 \%$ & $98,9 \%$ & $97,4 \%$ & $55,8 \%$ \\
\hline USP-BR-463-7 & $100,0 \%$ & $98,9 \%$ & $97,4 \%$ & $55,8 \%$ \\
\hline USP-BR-463-8 & $100,0 \%$ & $98,9 \%$ & $97,4 \%$ & $55,8 \%$ \\
\hline USP-BR-463-9 & $100,0 \%$ & $98,9 \%$ & $97,4 \%$ & $55,8 \%$ \\
\hline USP-BR-463-10 & $100,0 \%$ & $98,9 \%$ & $97,4 \%$ & $55,8 \%$ \\
\hline USP-BR-463-11 & $100,0 \%$ & $98,9 \%$ & $97,4 \%$ & $55,8 \%$ \\
\hline USP-BR-463-12 & $100,0 \%$ & $98,9 \%$ & $97,4 \%$ & $55,8 \%$ \\
\hline USP-BR-463-13 & $100,0 \%$ & $98,9 \%$ & $97,4 \%$ & $55,8 \%$ \\
\hline USP-BR-463-14 & $100,0 \%$ & $98,9 \%$ & $97,4 \%$ & $55,8 \%$ \\
\hline USP-BR-463-15 & $100,0 \%$ & $98,9 \%$ & $97,4 \%$ & $55,8 \%$ \\
\hline USP-BR-463-16 & $100,0 \%$ & $98,9 \%$ & $97,4 \%$ & $55,8 \%$ \\
\hline USP-BR-463-17 & $100,0 \%$ & $98,9 \%$ & $97,4 \%$ & $55,8 \%$ \\
\hline USP-BR-463-18 & $100,0 \%$ & $98,9 \%$ & $97,4 \%$ & $55,8 \%$ \\
\hline USP-BR-463-19 & $100,0 \%$ & $98,9 \%$ & $97,4 \%$ & $55,8 \%$ \\
\hline USP-BR-465-1 & $100,0 \%$ & $98,9 \%$ & $97,4 \%$ & $55,8 \%$ \\
\hline USP-BR-465-3 & $100,0 \%$ & $98,9 \%$ & $97,4 \%$ & $55,8 \%$ \\
\hline USP-BR-465-5 & $100,0 \%$ & $98,9 \%$ & $97,4 \%$ & $55,8 \%$ \\
\hline USP-BR-465-6 & $100,0 \%$ & $98,9 \%$ & $97,4 \%$ & $55,8 \%$ \\
\hline USP-BR-465-7 & $100,0 \%$ & $98,9 \%$ & $97,4 \%$ & $55,8 \%$ \\
\hline USP-BR-465-8 & $100,0 \%$ & $98,9 \%$ & $97,4 \%$ & $55,8 \%$ \\
\hline USP-BR-465-9 & $100,0 \%$ & $98,9 \%$ & $97,4 \%$ & $55,8 \%$ \\
\hline USP-BR-465-11 & $100,0 \%$ & $98,9 \%$ & $97,4 \%$ & $55,8 \%$ \\
\hline USP-BR-465-13 & $100,0 \%$ & $98,9 \%$ & $97,4 \%$ & $55,8 \%$ \\
\hline USP-BR-465-16 & $100,0 \%$ & $98,9 \%$ & $97,4 \%$ & $55,8 \%$ \\
\hline USP-BR-465-17 & $100,0 \%$ & $98,9 \%$ & $97,4 \%$ & $55,8 \%$ \\
\hline USP-BR-465-18 & $100,0 \%$ & $98,9 \%$ & $97,4 \%$ & $55,8 \%$ \\
\hline USP-BR-465-19 & $100,0 \%$ & $98,9 \%$ & $97,4 \%$ & $55,8 \%$ \\
\hline USP-BR-529-1 & $100,0 \%$ & $98,9 \%$ & $97,4 \%$ & $55,8 \%$ \\
\hline USP-BR-529-3 & $100,0 \%$ & $98,9 \%$ & $97,4 \%$ & $55,8 \%$ \\
\hline USP-BR-529-4 & $100,0 \%$ & $98,9 \%$ & $97,4 \%$ & $55,8 \%$ \\
\hline USP-BR-529-5 & $100,0 \%$ & $98,9 \%$ & $97,4 \%$ & $55,8 \%$ \\
\hline USP-BR-529-6 & $100,0 \%$ & $98,9 \%$ & $97,4 \%$ & $55,8 \%$ \\
\hline USP-BR-604-7 & $100,0 \%$ & $98,9 \%$ & $97,4 \%$ & $55,8 \%$ \\
\hline USP-BR-604-8 & $100,0 \%$ & $98,9 \%$ & $97,4 \%$ & $55,8 \%$ \\
\hline USP-BR-604-14 & $100,0 \%$ & $98,9 \%$ & $97,4 \%$ & $55,8 \%$ \\
\hline Vaccine-1 & $100,0 \%$ & $98,9 \%$ & $97,4 \%$ & $55,8 \%$ \\
\hline Vaccine-2 & $100,0 \%$ & $98,9 \%$ & $97,4 \%$ & $55,8 \%$ \\
\hline Vaccine-3 & $100,0 \%$ & $98,9 \%$ & $97,4 \%$ & $55,8 \%$ \\
\hline Vaccine-4 & $100,0 \%$ & $98,9 \%$ & $97,4 \%$ & $55,8 \%$ \\
\hline Calnek & ID & $98,9 \%$ & $97,4 \%$ & $55,8 \%$ \\
\hline Van Roekel & $95,2 \%$ & ID & $98,4 \%$ & $55,8 \%$ \\
\hline L2Z & $95,2 \%$ & $98,8 \%$ & $\mathrm{ID}$ & $54,3 \%$ \\
\hline HM 175 & $60,1 \%$ & $58,7 \%$ & $58,5 \%$ & ID \\
\hline
\end{tabular}

NUCLEOTIDE SEQUENCES 
BR-463-12 (KY292320), USP-BR-463-13 (KY292321), USP-BR-463-14 (KY292322), USP-BR-463-15 (KY292323), USP-BR-463-16 (KY292324), USPBR-463-17 (KY292325), 463-18 (KY292326), USPBR-463-19 (KY292327), USP-BR-465-1 (KY292328), USP-BR-465-3 (KY292329), USP-BR-465-5 (KY292330), USP-BR-465-6 (KY292331), USPBR-465-7 (KY292332), USP-BR-465-8 (KY292333), USP-BR-465-9 (KY292334), USP-BR-465-11 (KY292335), USP-BR-465-13 (KY292336), USPBR-465-16 (KY292337), USP-BR-465-17 (KY292338), USP-BR-465-18 (KY292339), USP-BR-465-19 (KY292340), USP-BR-529-1 (KY292341), USPBR-529-3 (KY292342), USP-BR-529-4 (KY292343), USP-BR-529-5 (KY292344),USP-BR-529-6 (KY292345), USP-BR-604-7 (KY292346), USP-BR-604-8 (KY292347) e USP-BR-604-14 (KY292348). The phylogenetic analysis (Figure 1) clustered all the samples together with

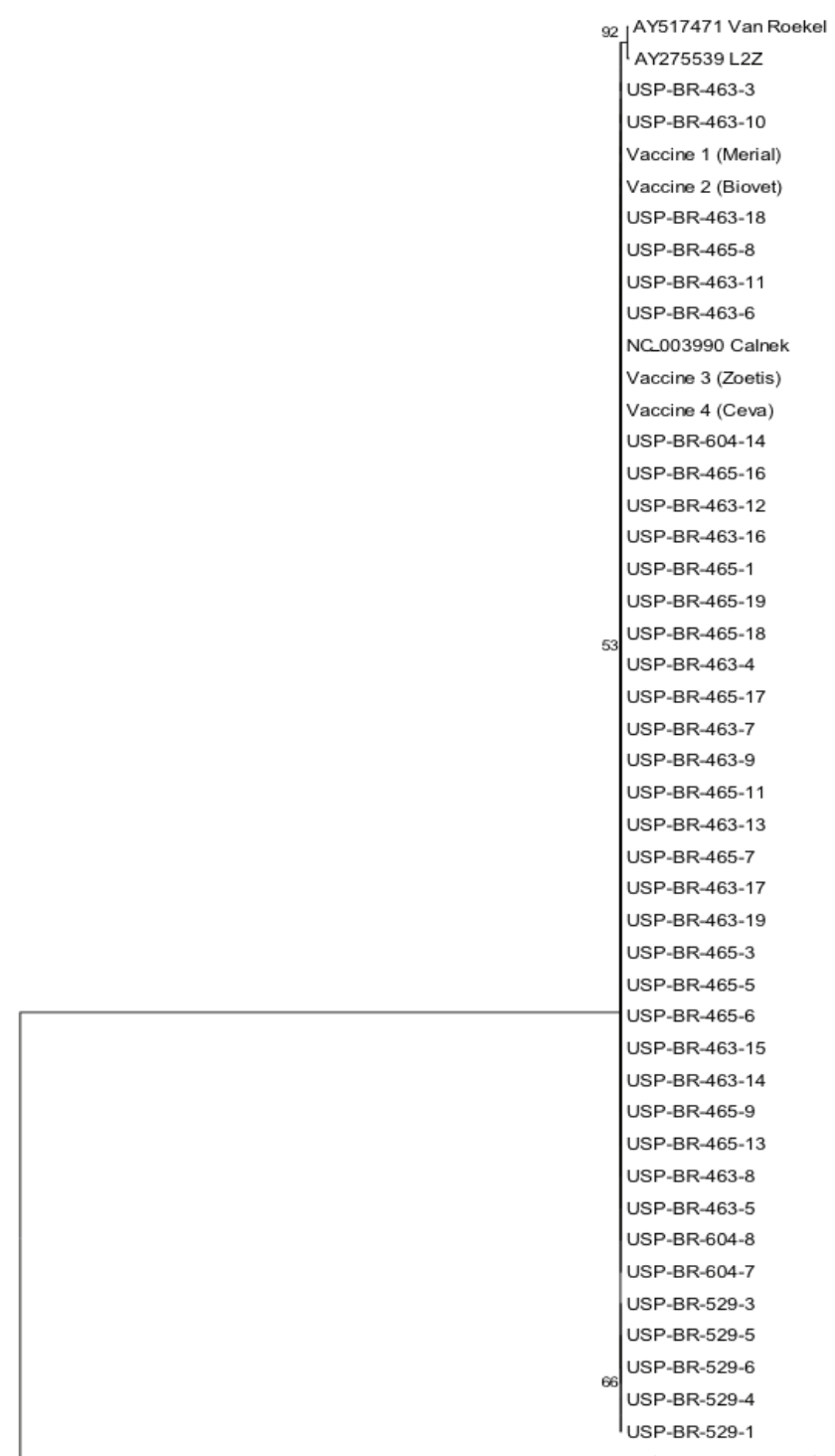

the four vaccines and the Calnek, Van Roekel and L2K strains, and in a different group, the HM 175 (Hepatovirus $A$ ) to show the difference between these two genera of the family Picornaviridae.

\section{DISCUSSION}

Avian encephalomyelitis is a disease distributed worldwide (Tannock \& Shafren, 1994; Boros et al., 2014; Haryanto et al., 2016; Welchman et al., 2009) and it has been described in Brazil in the state of Rio de Janeiro in 1692 (Chagas et al., 1962) and in the state of São Paulo in 1964 (Bueno et al., 1964). In 2015, an increase in cases of Avian Encephalomyelitis was reported; they were diagnosed through histopathology and serological analysis in the southern region of Brazil between 2012 and 2013 (Freitas \& Back, 2015). The results obtained in this study, confirm those of Freitas and Back (Freitas \& Back, 2015), finding the Tremovirus A in Avian Encephalomyelitis outbreaks, but using molecular techniques as diagnostic tools. The Avian Encephalomyelitis associated to Tremovirus $A$ is a disease that affects the nervous system in Young birds causing ataxia, weakness, tremors in the head and neck (Tannock \& Shafren, 1994), resulting in economic loss for poultry farmers due to a dropin food consumption and consequent decline in meat conversion (Boros et al., 2014). The highest susceptibility to this virus, where the clinical signs occurs, are the two first weeks of life (Suarez, 2013; Tannock \& Shafren, 1994), which support our findings due to the age of the birds tested with positive results that were not more than 15 days old for 41 samples, and 5 with no age revealed. The main symptom of Avian Encephalomyelitis is the tremor in the head and neck caused by a disorder in the nervous system (Boros et al., 2014), so, these organs are routinely tested for diagnosis of this disease. In this study, in addition to finding the virus in $39.1 \%$ of the brains and $71.4 \%$ of the sciatic nerves, the virus was also found in the digestive, respiratory, immune and renal systems. Thus, in addition to the organs of the nervous system, the digestive organs were also important for the diagnostic of Tremovirus A confirming the reports described by Tannock \& Shafren (1994) because in this study, Tremovirus A was detected in $36.8 \%$ of the intestines, $41,7 \%$ of gizzards, $71.4 \%$ of proventriculus, $57.1 \%$ of livers and $50 \%$ of cecum. The virus was also found in $40 \%$ of lungs, $40 \%$ of kidneys, $50 \%$ of bursas and $33.3 \%$ of thymus, demonstrating the possibility of finding this virus in these organs during outbreaks of $A E$. Although the infections by Tremovirus $A$ do 
not show clinical signs that affect the health of adult animals, they represent an economic risk for poultry farmers because there is a decrease in egg production (Yu et al., 2015), decrease in the number of births and increase of embryonic mortality in the case of breeder infections (Taylor et al., 1955). Another factor to be considered is that the progeny derived from these infected breeders, will have neurological signs associated with Avian Encephalomyelitis in the first weeks of life (Calnek, 1998). The results obtained in this study suggest a possible failure in the vaccination procedures in breeders, either due to errors in the management of the vaccine, poor storage conditions, changes in the vaccination schedule or delay in vaccine application with possible viral inactivation. In the other hand, it is important to emphasize that thanks to the vaccination of the breeders, the transfer of antibodies to the progeny occurs (Westbury \& Sinkovic, 1978), and that failures in vaccination procedures will decrease antibodies concentration in the progeny, and if they are challenged with a field virus, the infection will affect the chicks. Another hypothesis to be considered is that breeders were not vaccinated, or they got the field virus during the laying stage due to biosafety failures. Through analysis of the amino acid sequences, $100 \%$ of the samples had similarity to the vaccines and the reference strain (Calnek). The phylogenetic tree confirmed the data of the similarity matrix, clustering all the sequences within the same group of reference strains of Tremovirus A. The strain MH 175 was placed in a different group because it corresponds to a distinct genus called Hepatovirus (Knowles et al., 2012), which would explain one of the reasons for Tremovirus A to be withdrawn from the genus Hepatovirus for the new genus Tremovirus A (Knowles et al., 2012). Finally, all the viruses found in the samples correspond to the same molecular pattern of Tremovirus A. Probably, a failure occurred in the biosecurity procedure for preventing the outbreaks of $A E$ in progeny, due to the practice of vaccination nearby laying phase.

\section{CONCLUSIONS}

Tremovirus A presented the same molecular pattern of four vaccines used as control in this study. Considering the management of vaccine delivery to protect against Avian Encephalomyelitis, biosecurity measurements should be considered as a primordial pathway to avoid outbreaks in the progeny associated with vaccination programs as the best strategy for controlling AE outbreaks in chicken production.

\section{ACKNOWLEDGMENTS}

The authors are immensely grateful for the support from CNPq grant \#453920/2014-4. A. J. Piantino Ferreira is also a recipient of CNPq fellowships. Also, we would like to thank the poultry companies who sent biological samples for this study.

\section{REFERENCES}

Bakhshesh M, Groppelli E, Willcocks MM, Royall E, Belsham GJ, Roberts LO. The picornavirus avian encephalomyelitis virus possesses a hepatitis $C$ virus-like internal ribosome entry site element. Journal of Virology 2008;82:1993-2003

Boros Á, Pankovics P, Reuter G. Avian picornaviruses : Molecular evolution, genome diversity and unusual genome features of a rapidly expanding group of viruses in birds. Infection, Genetic and Evolution 2014;28:151166.

Bueno RC, Baquer SR, Nakano M. Ocorrência da encefalo-mielite aviária em São Paulo. Isolamento e identificação do vírus responsável. Revista Brasileira de Biologia 1964;24:31-44.

Calnek BW. Control of avian encephalomyelitis : a historical account. Avian Diseases 1998;42:632-647.

Chomczynski P. Single-step method of rna isolation by acid guanidinium extraction. Analytical Biochemistry1987;162:156-159.

Chagas AW, Dacorso Filho PJ, Vaitsmann J. Encefalo-mielite aviária. Primeira identificação nos Estados da Guanabara e Rio de Janeiro. Revista de Veterinaria 1962;15:8-10

Freitas ES, Back A. New occurance of avian encephalomyelitis in broiler - is this an emerging disease? Brazilian Journal of Poultry Science 2015;17:399-404.

Gosting LH, Grinnell BW, Matsumoto M. Physico-chemical and morphological characteristics of avian encephalomyelitis virus. Veterinary Microbiology 1980;5:87-100.

Haryanto A, Ermawati R, Wati V, Irianingsih SH, Wijayanti N. Analysis of viral protein-2 encoding gene of avian encephalomyelitis virus from field specimens in Central Java region, Indonesia. Veterinary World 2016;9:25-31.

Ikeda S, Matsuda K, Yonaiyama K. Susceptibility of chickens to avian encephalomyelitis virus. III. Behavior of the virus in growing chicks. National Institute of Animal Health Q 1976;16:33-38.

Jones E. An encephalomyelitis in chicken. Science 1932;76:331-331.

Knowles NJ, Hovi T, Hyypia T, King AMQ, Lindberg AM, et al. Family picornaviridae. In: King AMQ, Adams MJ, Carstens EB, Lefkowitz EJ, editors. Virus taxonomy: classification and nomenclature of viruses: nineth report of the international committee on taxonomy of viruses. London: Elsevier; 2012. p.855-880.

Marvil P, Knowles NJ, Mockett APA, Britton P, Brown TDK, Cavanagh D Avian encephalomyelitis virus is a picornavirus and is most closely related to hepatitis A virus. Journal of GeneralVirology 1999; 80:653662.

Olitsky PK. Experimental studies on the virus of infectious avian encephalomyelitis. Journal of Experimental Medicine 1939;70:565582 .

Suarez DL. Avian Encephalomyelitis. In: Swayne DE, Glisson JR, McDougald LR, Nolan LK, Suarez DL, Nair V, editors. Diseases of poultry. Ames: Wiley-Blacwell; 2013. p.486-493. 
Tamura K, Peterson D, Peterson N, Stecher G, Nei M, Kumar S. MEGA 5: molecular evolutionary genetics analysis using maximum likelihood, evolutionary distance, and maximum parsimony methods. Molecular Biology and Evolution 2011;28:2731-2739.

Tannock GA, Shafren DR. Avian encephalomyelitis: a review. Avian Pathology 1994;23:603-620.

Taylor LW, Lowry DC, Raggi LG. Effects ofan outbreak of avian encephalomyelitis (epidemic tremor) in a breeding flock. Poultry Science 1955;34:1036-1045.

Welchman, DB, Cox WJ, Gough RE, Wood AM, Smyth VJ, Todd D, et al. Avian encephalomyelitis virus in reared pheasants : a case study. Avian Pathology 2009; 38:251-256.
Westbury HA Sinkovic B. The Pathogenesis of infectious avian encephalomyelitis 4. The effect of maternal antibody on the development of the disease. Australian Veterinary Journal 1978;54:8185.

Xie Z, Khan MI, Girshick T, Xie Z. Reverse transcriptase-polymerase chain reaction to detect avian encephalomyelitis virus. Avian Diseases 2005;49:227-230

Yu XH, Zhao J, Qin XH, Zhang GZ. Serological evidence of avian encephalomyelitis virus infection associated with vertical transmission in chicks. Biologicals 2015;43:512-514. 
